\title{
Mixed Time/Frequency-Domain Based Robust Identification
}

\author{
Pablo A. Parrilo1 2, Mario Sznaier ${ }^{3}$, Ricardo S. Sánchez Peña *
}

\begin{abstract}
In this paper we propose a new robust identification framework that combines both frequency and time-domain experimental data. The main result of the paper shows that the problems of establishing consistency of the data and of obtaining a nominal model and bounds on the identification error can be recast as a constrained finite-dimensional convex optimization problem that can be efficiently solved using Linear Matrix Inequalities techniques. This approach, based upon a generalized interpolation theory, contains as special cases the Carathéodory-Fejér (purely time-domain) and Nevanlinna-Pick (purely frequency-domain) problems. The proposed procedure interpolates the frequency and time domain experimental data while restricting the identified system to be in an a priori given class of models, resulting in a nominal model consistent with both sources of data. Thus, it is convergent and optimal up to a factor of 2 (with respect to central algorithms).
\end{abstract}

\section{Introduction}

During the past few years a large research effort has been devoted to the problem of developing deterministic identification procedures that, starting from experimental data and an a priori class of models, generate a nominal model and bounds on identification errors. These models and bounds can then be combined with standard robust control synthesis methods (such as $\mathcal{H}_{\infty}, \mu$ or $\ell^{1}$ ) to obtain robust systems. This problem, termed the Robust Identification problem was originally posed by Helmicki et. al. [7] and has since attracted considerable attention $[3,6,8,9,12,14,16,17]$ and references therein.

The case where the experimental data available is generated by frequency-domain experiments leads to $\mathcal{H}_{\infty}-$ based identification procedures. In this context the main effort has been directed towards establishing robust convergence of the algorithms and analyzing their untuned characteristics [6].

\footnotetext{
${ }^{1}$ Depto. de Electrónica, Facultad de Ingeniería, Universidad de Buenos Aires, Argentina.

${ }^{2}$ Becario de Iniciación del Programa de Informática, U.B.A. email: pparrilQaleph.fi.uba.ar

${ }^{3}$ Dept. of Electrical Engineering, Penn State University, University Park, PA 16802. email: msznaier Qfrodo.ee.psu.edu.

${ }^{4}$ National Commission of Space Activities (CONAE), Argentina. email: ricardo@orbis.conae.gov.ar

0-7803-3590-2/96 \$5.00 @ 1996 IEEE
}

The case where the experimental data available originates from time-domain experiments leads to $\ell_{1}$ identification, addressed in $[8,10,12]$. The untuned nature of the algorithms based on time series is strongly dependent on the input sequence [10]. It can be shown that there is no untuned algorithm capable of identifying a system using only impulse response measurements [8]. Finally, recent papers $[5,18]$ proposed interpolatory algorithms that use data obtained from time domain experiments to generate a nominal model together with an $\mathcal{H}_{\infty}$ bound on the identification error.

In this paper we propose a new robust identification framework that takes into account both time and frequency domain experiments. Thus, the problem where "good" frequency response fitting (small $\mathcal{H}_{\infty}$ error norm) leads to "poor" fitting in the time-domain is avoided. Additionally, from an information theoretic viewpoint, more experiments produce a smaller consistency set of undistinguishable models, and as a consequence a smaller worst case error.

The main result of the paper shows that the problems of establishing consistency of the data and of obtaining a nominal model and bounds on the identification error can be recast as a constrained finite-dimensional convex optimization problem that can be efficiently solved using Linear Matrix Inequalities techniques. This approach includes as special cases the frequency based approach of Chen et. al. [3] and the time domain approach of Chen and Nett [5] and Zhou and Kimura [18].

\section{Preliminaries}

\subsection{Notation}

By $\mathcal{L}_{\infty}$ we will denote the Lebesgue space of complex valued matrix functions essentially bounded on the unit circle, equipped with the norm:

$$
\|G(z)\|_{\infty} \triangleq e s s \sup _{|z|=1} \bar{\sigma}(G(z))
$$

where $\bar{\sigma}$ denotes the largest singular value. By $\mathcal{H}_{\infty}$ we denote the subspace of functions in $\mathcal{L}_{\infty}$ with a bounded analytic continuation inside the unit disk, equipped with the norm $\|G(z)\|_{\infty} \triangleq$ ess $\sup _{|z|<1} \bar{\sigma}(G(z))$. Also of interest is the space $\mathcal{H}_{\infty, p}$ of transfer matrices in $\mathcal{H}_{\infty}$ which have analytic continuation inside the disk of ra- 
dius $\rho>1$, i.e. the space of exponentially stable systems with a stability margin of $(\rho-1)$. When equipped with the norm $\|G(z)\|_{\infty, \rho} \triangleq \sup _{|z|<\rho} \bar{\sigma}(G(z)), \mathcal{H}_{\infty, \rho}$ becomes a normed Banach space. $\overline{\mathcal{B}} \mathcal{H}_{\infty} \triangleq\left\{F \in \mathcal{H}_{\infty}\right.$, $\left.\|F\|_{\infty} \leq 1\right\}$ denotes the closed unit-ball in $\mathcal{H}_{\infty}$. Similarly $\overline{\mathcal{B}} \mathcal{H}_{\infty, \rho}$ denotes the closed unit-ball in $\mathcal{H}_{\infty, \rho}$.

Given a vector $x \in R^{n}$ its infinity norm is defined as $\|x\|_{\infty} \triangleq \max _{i}\left|x_{i}\right| . \ell_{1}$ denotes the space of absolutely summable sequences $h=\left\{h_{i}\right\}$ equipped with the norm $\|h\|_{\ell_{1}} \triangleq \sum_{i=0}^{\infty}\left|h_{i}\right|<\infty$. $\ell_{\infty}$ denotes the space of bounded sequences $h=\left\{h_{i}\right\}$ equipped with the norm $\|h\|_{\ell_{\infty}} \triangleq$ sup $\left|h_{i}\right|<\infty$. Given a sequence $h \in \ell_{1}$, its $z$-transform $i \geq 0$

is defined as $H(z)=\sum_{i=0}^{\infty} h_{i} z^{i 1}$.

For simplicity in the sequel we consider SISO models, although all results can be applied to MIMO systems, following Chen et. al. [4].

\subsection{The Robust Identification Framework} In this paper we consider the case where the a posteriori experimental data originates from two different sources: i) frequency and ii) time domain experiments. The first type of information consists of a set of $N_{f}$ samples of the frequency response of the system: $y_{k}^{f}=\hat{h}_{k}+\eta_{k}^{f}, k=$ $0, \ldots, N_{f}-1$, where $\hat{h}_{k}=H\left(e^{j \Omega_{k}}\right), \quad k=0, \ldots, N_{f}$ $1, \Omega_{k}$ denotes the sampling frequencies; and where $\eta_{k}^{f}$ represents complex additive noise, bounded by $\epsilon_{f}$ in the $\ell_{\infty}$ norm (i. e. in the class $\ell_{\infty}\left(\epsilon_{f}\right)$ ).

The time domain data consists of a set of the first $N_{t}$ samples of the time response corresponding to a known but otherwise arbitrary input, also corrupted by additive noise $y_{n}^{t}=(U h)_{n}+\eta_{n}^{t}, n=0, \ldots, N_{t}-1$, where

$$
U=\left[\begin{array}{cccc}
u_{0} & 0 & \ldots & 0 \\
u_{1} & u_{0} & \ldots & 0 \\
\vdots & \ddots & \ddots & 0 \\
u_{N_{t}-1} & \ldots & u_{1} & u_{0}
\end{array}\right]
$$

is the Toeplitz matrix corresponding to the input sequence and where the noise $\eta_{n}^{t}$ is real and belongs to $\ell_{\infty}\left(\epsilon_{t}\right)$. In the sequel, for notational simplicity we will collect the samples $y_{k}^{f}$ and $y_{n}^{t}$ in the vectors $\mathbf{y}^{f} \in \mathbb{C}^{N_{f}}$ and $\mathrm{y}^{t} \in \mathcal{R}^{N_{s}}$.

The a priori information available is that the system $H$ under consideration belongs to the following classes of models:

1. $H \in \mathcal{H}_{\infty}(\rho, K) \triangleq\left\{H \in \mathcal{H}_{\infty, \rho}:\|H\|_{\infty, \rho} \leq K\right\}$ i.e. exponentially stable systems having a stability margin of $(p-1)$ and a peak response to complex exponential inputs of $K$. Thus the impulse response of these systems satisfies the following

${ }^{1}$ Note that this is the inverse of the usual $z$ transform. Therefore for causal, stable systems $H(z)$ is analytical in $|z|<1$. time domain bound:

$$
|h(k)| \leq K \rho^{-k}
$$

2. Additionally, the system $H$ is known to belong to a class $\Phi$ of models satisfying a time-domain bound of the form:

$\Phi \triangleq\left\{h(\cdot) \mid \phi_{\ell}(k) \leq h(k) \leq \phi_{u}(k), \quad k=0, N_{\phi}-1\right\}$

Note that this class includes as a special case the systems described by (1) when $\phi_{\ell}(k)=-K \rho^{-k}$ and $\phi_{u}(k)=K \rho^{-k}$.

To combine both classes of models we define the a priori set of systems

$$
\mathcal{S} \triangleq \Phi \cap \mathcal{H}_{\infty}(\rho, K)
$$

The a priori information we have considered simply adds to the usual $\mathcal{H}_{\infty}$ identification procedures a bound on the first $N_{\phi}$ samples of the impulse response.

To recap, the a priori information and the a posteriori experimental input data are:

$$
\begin{aligned}
\mathcal{S} & =\Phi \cap \mathcal{H}_{\infty}(\rho, K), \quad(\rho>1, K<\infty) \\
\mathcal{N}_{f} & =\ell_{\infty}\left(\epsilon_{f}\right)=\left\{\eta^{f} \in \mathbb{C}^{N_{f}}, \quad\left|\eta_{k}^{f}\right| \leq \epsilon_{f}\right\} \\
\mathcal{N}_{t} & =\ell_{\infty}\left(\epsilon_{t}\right)=\left\{\eta^{t} \in \mathcal{R}^{N_{t}}, \quad\left|\eta_{k}^{t}\right| \leq \epsilon_{t}\right\} \\
\mathbf{y}^{f} & =\left\{\hat{h}+\eta^{f} \in \mathbb{C}^{N_{f}}\right\} \\
\mathbf{y}^{t} & =\left\{\left[(U h)_{j}+\eta_{j}^{t}\right]_{0, N_{t}-1} \in \mathcal{R}^{N_{t}}\right\}
\end{aligned}
$$

By using these definitions the robust identification problem with mixed data can be precisely stated as:

Problem 1 Given the experiments $\left(\mathrm{y}^{f}, \mathrm{y}^{t}\right)$ and the a priori sets $\left(\mathcal{S}, \mathcal{N}_{f}, \mathcal{N}_{t}\right)$, determine:

1. If the a priori and a posteriori information are consistent, i.e. the consistency set

$$
\mathcal{S}\left(\mathbf{y}^{f}, \mathbf{y}^{t}\right) \triangleq\left\{\begin{array}{l|l}
H \in \mathcal{S} & \begin{array}{l}
\left(\mathbf{y}^{f}-\hat{h}\right) \in \mathcal{N}_{f} \\
\left(\mathbf{y}^{t}-U h\right) \in \mathcal{N}_{t}
\end{array}
\end{array}\right\}
$$

is nonempty.

2. If (3) holds, find a nominal model in the consistency set $\mathcal{S}\left(\mathrm{y}^{f}, \mathrm{y}^{t}\right)$, and an error bound.

\subsection{Generalized Interpolation Framework} In this section we briefly present a generalized interpolation framework developed in [1] and applied to $\mathcal{H}_{\infty}$ control in [15]. This framework will be used in section III to solve Problem 1

Theorem 1 There exists a transfer function $f(z) \in$ $\mathcal{B} \mathcal{H}_{\infty}\left(\overline{\mathcal{B}} \mathcal{H}_{\infty}\right)$ such that:

$$
\sum_{z_{0} \in \mathcal{D}} \operatorname{Res}_{z_{z}=z_{0}} f(z) C_{-}(z I-A)^{-1}=C_{+}
$$


if and only if the following discrete time Lyapunov equation has a unique positive (semi) definite solution.

$$
M=A^{*} M A+C_{-}^{*} C_{-}-C_{+}^{*} C_{+}
$$

where $A, C_{-}$and $C_{+}$are constant complex matrices of appropriate dimensions. If $M>0$ then the solution $f(z)$ is non-unique and the set of solutions can be parametrized in terms of $q(z)$, an arbitrary element of $\bar{B} \mathcal{H}_{\infty}$, as follows:

$$
\begin{aligned}
& f(z)=\frac{T_{11}(z) q(z)+T_{12}(z)}{T_{21}(z) q(z)+T_{22}(z)} \\
& T(z)=\left[\begin{array}{ll}
T_{11}(z) & T_{12}(z) \\
T_{21}(z) & T_{22}(z)
\end{array}\right]
\end{aligned}
$$

where $T(z)$ is the $J$-lossless ${ }^{2}$ matrix:

$$
\begin{aligned}
T(z) & \equiv\left[\begin{array}{l|l}
A_{T} & B_{T} \\
\hline C_{T} & D_{T}
\end{array}\right] \\
A_{T} & =A \\
B_{T} & =M^{-1}\left(A^{*}-I\right)^{-1}\left[\begin{array}{ll}
-C_{+}^{*} & C_{-}^{*}
\end{array}\right] \\
C_{T} & =\left[\begin{array}{l}
C_{+} \\
C_{-}
\end{array}\right](A-I) \\
D_{T} & =I+\left[\begin{array}{l}
C_{+} \\
C_{-}
\end{array}\right] M^{-1}\left(A^{*}-I\right)^{-1}\left[\begin{array}{ll}
-C_{+}^{*} & C_{-}^{*}
\end{array}\right]
\end{aligned}
$$

Remark 1 It can be shown that both the NevanlinnaPick and the Carathéodory-Fejér problems are special cases of this theorem, corresponding to an appropriate choice of the matrices $A$ and $C_{-}$[15].

\section{Main Results}

Nevanlinna-Pick based identification algorithms address the case where the experimental data available is purely frequency domain, while Carathéodory-Fejér -based identification deals only with time domain data. In this section we exploit the generalized interpolation framework introduced in the previous section to solve Problem 1, obtaining a robust identification algorithm that combines both sources of data. To this effect, we will divide Problem 1 into two subproblems: i) consistency and ii) identification. The first consists of determining the existence of a candidate model $H \in \mathcal{S}$ which may have produced both, the time and frequency domain experimental data. Clearly, this is a prerequisite to the second stage, the computation of the nominal model itself and a bound on the identification error.

\subsection{Consistency}

From equation (3) it follows that the problem of determining consistency of the a posteriori and a priori information reduces to establishing whether or not there

\footnotetext{
${ }^{2}$ A transfer function $H(z)$ is said to be $J$-lossless if $H^{T}(1 / z) J H(z)=J$ when $|z|=1$, and $H^{T}(1 / z) J H(z)<J$ when $|z|<1$. Here $J=\left[\begin{array}{cc}I & 0 \\ 0 & -I\end{array}\right]$.
}

exists a model $H \in \mathcal{S}$ that interpolates the frequency experimental data:

$$
\hat{h}=\mathbf{y}^{f}+\eta^{f}, \quad \eta^{f} \in \mathcal{N}_{f}
$$

and has an impulse response that satisfies the following constraints:

$$
U h=\mathbf{y}^{t}+\eta^{t}, \quad \eta^{t} \in \mathcal{N}_{t}
$$

where the noiseless output $U h$ is the convolution of the input vector $u^{T}=\left[\begin{array}{llll}u_{0} & u_{1} & \ldots & u_{N_{t}-1}\end{array}\right]$ and the system $H(z)$.

The main result of this section shows that consistency can be established by solving a finite-dimensional convex optimization problem. To establish this result we will first obtain an equivalent condition for consistency (Lemma 1) in the form of a linearly constrained generalized interpolation problem. In Theorems 2 and 3 we will show that this generalized problem can be recast in terms of an LMI optimization.

Lemma 1 The a priori and a posteriori information are consistent if and only if there exists a function $H \in$ $\mathcal{H}_{\infty}(\rho, K)$ such that

$$
\begin{aligned}
& \hat{h}=\mathbf{y}^{f}+\eta^{f}, \quad \eta^{f} \in \mathcal{N}_{f} \\
& \mathbf{y}_{L} \leq\left[\begin{array}{l}
U \\
I
\end{array}\right] h \leq \mathbf{y}_{U}
\end{aligned}
$$

where

$$
\mathbf{y}_{L}=\left[\begin{array}{c}
\mathbf{y}_{1}^{t}-\epsilon_{t} \\
\vdots \\
\mathbf{y}_{N_{t}-1}^{t}-\epsilon_{t} \\
\phi_{l}(0) \\
\vdots \\
\phi_{\ell}\left(N_{\phi}-1\right)
\end{array}\right] \quad, \quad \mathbf{y}_{U}=\left[\begin{array}{c}
\mathbf{y}_{1}^{t}+\epsilon_{t} \\
\vdots \\
y_{N_{t}-1}^{t}+\epsilon_{t} \\
\phi_{u}(0) \\
\vdots \\
\phi_{u}\left(N_{\phi}-1\right)
\end{array}\right]
$$

The next Theorem provides necessary and sufficient conditions for the existence of a function $H \in$ $\mathcal{H}_{\infty}(\rho, K)$ which interpolates fixed frequency domain experimental data while, at the same time, satisfying a time-domain constraint.

Theorem 2 Given $N_{f}$ frequency-domain data points, $H\left(z_{i}\right)=w_{i}, \quad i=0, \ldots, N_{f}-1$ and $N_{t}$ timedomain data points $h_{k}, k=0, \ldots, N_{t}-1$, there exists $H \in \mathcal{H}_{\infty}(\rho, K)$ that interpolates the frequency domain data and such that $H(z)=h_{0}+h_{1} z+h_{2} z^{2}+\ldots+$ $h_{N_{t}-1} z^{N_{t}-1}+\ldots$ if and only if

$$
\begin{aligned}
& M_{R}(\mathrm{w}, h) \triangleq \\
& {\left[\begin{array}{cc}
Q-\frac{1}{K^{2}} \mathcal{W}_{f}^{*} Q \mathcal{W}_{f} & M_{X} \\
M_{X}^{*} & R^{-2}-\frac{1}{K^{2}} \mathcal{F}_{t}^{*} R^{-2} \mathcal{F}_{t}
\end{array}\right]>0}
\end{aligned}
$$


where

$$
\begin{aligned}
& M_{X}=S_{0} R^{-2}-\frac{1}{K^{2}} \mathcal{W}_{f}^{*} S_{0} R^{-2} \mathcal{F}_{t} \\
& R=\operatorname{diag}\left[\begin{array}{ccccc}
1 & \rho & \rho^{2} & \ldots & \rho^{N_{t}-1}
\end{array}\right] \\
& Q=\left[\frac{\rho^{2}}{\rho^{2}-\bar{z}_{i} z_{j}}\right]_{i j} \\
& S_{0}=\left[\begin{array}{ccccc}
1 & \bar{z}_{0} & \bar{z}_{0}^{2} & \ldots & \bar{z}_{0}^{N_{t}-1} \\
1 & \bar{z}_{1} & \bar{z}_{1}^{2} & \ldots & \bar{z}_{1}^{N_{t}-1} \\
\vdots & \vdots & \vdots & \vdots & \vdots \\
1 & \bar{z}_{N_{f}-1} & \bar{z}_{N_{f}-1}^{2} & \ldots & \bar{z}_{N_{f}-1}^{N_{t}-1}
\end{array}\right] \\
& \mathcal{W}_{f}=\operatorname{diag}\left[\begin{array}{lll}
w_{0} & \ldots & w_{N_{f}-1}
\end{array}\right] \\
& \mathcal{F}_{t}=\left[\begin{array}{cccc}
h_{0} & h_{1} & \ldots & h_{N_{t}-1} \\
0 & h_{0} & \ldots & h_{N_{i}-2} \\
\vdots & \vdots & \ddots & \vdots \\
0 & 0 & \ldots & h_{0}
\end{array}\right]
\end{aligned}
$$

Remark 2 The $(1,1)$ block of $M_{R}$ is the Pick matrix corresponding to the frequency domain consistency problem solved in Chen. et. al. [3] via the clessical Nevanlinna-Pick interpolation. Block $(2,2)$ is the Carathéodory-Fejér matrix corresponding to the time domain consistency problem solved in [5] and [4]. $M_{X}$ is a cross-coupling term due to the existence of both types of experimental data.

Combining the previous result with Lemma 1 yields the following necessary and sufficient condition for consistency:

Lemma 2 The a priori and a posteriori information are consistent if and only if there exists two vectors:

$$
\mathbf{w}=\left[\begin{array}{c}
w_{0} \\
w_{1} \\
\cdots \\
w_{N_{f}-1}
\end{array}\right] \quad, \quad h=\left[\begin{array}{c}
h_{0} \\
h_{1} \\
\cdots \\
h_{N_{t}-1}
\end{array}\right]
$$

such that

$$
\begin{aligned}
M_{R}(\mathbf{w}, h) & >0 \text { and } \\
\left(\mathbf{w}-\mathbf{y}^{f}\right) \in \mathcal{N}_{f} & , \quad\left(U h-\mathbf{y}^{t}\right) \in \mathcal{N}_{t}
\end{aligned}
$$

From Lemma 2 it follows that the consistency problem can be reduced to solving a feasibility problem in terms of the time and frequency domain vectors $h$ and $w$. This feasibility problem can be recast in terms of LMI's (and thus efficiently solved, using for instance interiorpoint methods $[13,2]$ ) as follows.

Theorem 3 The consistency problem with mixed time/frequency-domain data is equivalent to the follow- ing $L M I$ feasibility problem (in $\mathcal{F}_{t}$ and $\mathcal{W}$ ):

$$
\begin{aligned}
{\left[\begin{array}{cc}
M_{0}^{-1} & \frac{1}{K} X \\
\frac{1}{K} X^{*} & M_{0}
\end{array}\right]>0 } \\
{\left[\begin{array}{cc}
\epsilon_{f}^{2} I & \mathcal{W}-\mathcal{y}^{f} \\
\left(\mathcal{W}-\mathcal{Y}^{f}\right)^{*} & I
\end{array}\right]>0 } \\
-\epsilon_{t}<\mathcal{F}_{t}^{T} u-\mathbf{y}^{t}<\epsilon_{t}
\end{aligned}
$$

where this last inequality should be understood in the componentwise sense and where

$$
\begin{aligned}
M_{0} & =\left[\begin{array}{cc}
Q & S_{0} R^{-2} \\
R^{-2} S_{0}^{*} & R^{-2}
\end{array}\right] \\
X & =\left[\begin{array}{cc}
\mathcal{W}_{f} & 0 \\
0 & \mathcal{F}_{t}
\end{array}\right] \\
\mathcal{Y}_{f} & =\operatorname{diag}\left\{y^{f}\right\}
\end{aligned}
$$

\subsection{Identification}

Once consistency is established, the second step towards solving Problem 1 consists of generating a nominal model in the consistency set $\mathcal{S}\left(\mathbf{y}^{f}, \mathbf{y}^{t}\right)$. The identification algorithm that we propose is based on the parameterization of all solutions of the generalized Nevanlinna-Pick interpolation problem [1] presented in Theorem 1. For simplicity we consider the case where the matrix $M_{R}$ is strictly positive definite and therefore the solution is nonunique. Details for the degenerate case where there exists a unique solution can be found in [1]. The algorithm can be summarized as follows

1.- Find feasible data vectors $w, h$ for the consistency problem (21), (22) by solving the LMI feasibility problem given by (23).

2.- Compute the generalized Pick matrix $M_{R}$ in (13).

3.- Use Theorem 1 to compute a model from the consistency set $\mathcal{S}$. Recall that all the models in $\mathcal{S}$ (i.e. all the solutions to the generalized interpolation problem) can be parametrized as a Linear Fractional Transformation (LFT) of a free parameter $q(z) \in \overline{\mathcal{B}} \mathcal{H}_{\infty}$ as follows:

$$
\begin{aligned}
& H(z)=F_{\ell}[L(z), q(z)] \\
& L(z)=\left[\begin{array}{cc}
T_{12} T_{22}^{-1} & T_{11}-T_{12} T_{22}^{-1} T_{21} \\
T_{22}^{-1} & -T_{22}^{-1} T_{21}
\end{array}\right]
\end{aligned}
$$

In particular, if the free parameter $q(z)$ is chosen as a constant, then the model order is less than or equal to $N_{f}+N_{t}$.

Remark 3 Note that $T(z)$ depends on the choice of vectors $\mathrm{w}, h$. Thus, there are additional degrees of freedom available in the problem (choices of $w, h$ and $q(z)$ ) that could be used to optimize additional performance criteria (e.g. model order). 
Since the proposed algorithm is interpolatory, it has several advantages over the usual "two step" algorithms sometimes used in the context of robust identification $[6,7]$. In particular, since the identified model is in set $\mathcal{S}\left(\mathbf{y}^{f}, \mathbf{y}^{t}\right)$, its distance to the Chebyshev center of this set is within the diameter of information [11]. As a consequence the algorithm is optimal up to a factor of 2 as compared with central strongly optimal procedures. For the same reasons, it is also convergent and therefore the modelling error tends to zero as the information is completed.

\subsection{Analysis of the Identification Error}

In this section we derive upper and lower bounds for the worst-case identification error. Since these bounds are given in terms of the radius and diameter of information $[7,3]$, they are valid for all interpolatory algorithms taking as inputs the available a priori and a posteriori information.

Lemma 3 Assume that $\Phi_{u}(k)=-\Phi_{\ell}(k)=\Phi(k) \geq$ $0, k=0, \ldots, N_{\Phi}-1$ (symmetric time domain a priori information), and let $\hat{\beta}=\min \left[\epsilon_{f}, \Phi(0), \frac{\epsilon_{t}}{\|u\|<_{\infty}}\right]$, where $u$ is a vector whose components are the input signal sequence. Then, the radius of information $\mathcal{R}_{\mathcal{I}}$ satisfies:

$$
\begin{array}{ll}
\text { If } \hat{\beta} \geq K & , \quad \mathcal{R}_{\mathcal{I}} \geq K \\
\text { If } \hat{\beta}<K & , \quad \mathcal{R}_{\mathcal{I}} \geq \frac{K\|B(z)\|_{\infty}+\hat{\beta}}{1+\|B(z)\|_{\infty} \hat{\beta} / K}
\end{array}
$$

Lemma 4 Assume the same a priori information as in the previous lemma. Then the radius of information $\mathcal{R}_{\mathcal{I}}$ can be bounded above by:

$$
\mathcal{R}_{\mathcal{I}} \leq \sum_{i=0}^{M} \nu_{i}+\frac{K}{\rho^{M}(\rho-1)}
$$

where $M=N_{t}+N_{f}-1$ and $\nu_{i}$ are a function of the a priori information only.

\section{Example}

In this section, we present a simple example that il lustrates the importance of considering both time and frequency experimental information. Take the following data:

1. A priori information: $K=10, \rho=5$. For simplicity, we will initially consider $\epsilon_{f}=\epsilon_{t}=0$ (noiseless sampling).

2. A posteriori information:

- Frequency data: $\hat{f}(1)=1, \hat{f}(j)=1, \hat{f}(-1)=1$

- Time domain data: $f_{0}=1, f_{1}=0.1, f_{2}=0.01$
We will see that the a priori assumptions are consistent with the time domain or frequency domain a posteriori information, but not with both simultaneously. To this end, note that $g(z)=1$ belongs to $\mathcal{H}_{\infty}(\rho, K)$, and interpolates exactly the frequency data. On the other hand,

$$
h(z)=\frac{10}{10-z}
$$

also belongs to $\mathcal{K}_{\infty}(\rho, K)$, and interpolates exactly the time domain data. However, the generalized Pick matrix corresponding to this data is not positive definite, and therefore there is no function in $\mathcal{H}_{\infty}(\rho, K)$, that interpolates simultaneously both set of data.

Note in passing that in the noiseless case it is not necessary to use the generalized theory, as we can always find the solution to the "pure" Carathéodory-Fejér problem, and then find interpolation constraints on the free parameter $q(z)$. The real advantage of our procedure appears in practical cases with the presence of both, time and frequency measurements errors.

To see this, we will use our algorithm to compute the smallest noise bound ${ }^{3}$ that renders the experimental data consistent with the a priori information. In this example the smallest noise bound necessary for consistency satisfies $0.0484<\epsilon_{\text {min }}<0.0485$. This means that if (time and frequency) noise level is below 0.0484 the a posteriori and a priori information are inconsistent. On the other hand, if both (time and frequency) levels are above 0.0485 , there always exists an interpolating function for both types of data.

In the latter case, a transfer function in the a priori class that approximately interpolates the samples is given by

$$
f(z)=\frac{\sum_{i=0}^{7} n_{i} z^{i}}{\sum_{i=0}^{7} d_{i} z^{i}}
$$

where the coefficients $n_{i}, d_{i}$ are given in Table 1 . This function was obtained by taking $q(z)=0$, in the parameterization in Theorem 1 . Note that this function is analytical in $|\rho| \leq 5$ and that the supremum of $|f(z)|$ on $|z|<\rho$ is 9.983 , barely below $K=10$.

\section{Conclusions and Directions for Future Research}

In this paper we propose a new generalized robust identification framework that combines both frequency and time-domain experimental data, thus avoiding situations where a "good" fit of the data provided by one class of experiments (such as frequency domain) leads to poor fitting of the data provided by the other experiments. This situation was illustrated with the simple example of section IV, where the time and frequency

\footnotetext{
${ }^{3}$ For simplicity, we consider the time and frequency noise bounds to be equal. There is no diffeulty in removing this assumption.
} 


\begin{tabular}{|c|c|c|}
\hline$i$ & $\overline{n_{i}}$ & $d_{i}$ \\
\hline 7 & $9.999767740779586 e+001$ & $1.000000000000000 \mathrm{e}+000$ \\
\hline 6 & $5.072924867250237 e+000$ & $1.338280454587666 e+000$ \\
\hline 5 & $-2.551640808285396 e+003$ & $-2.545742554172364 e+001$ \\
\hline 4 & $-5.372773706720136 e+001$ & $-3.375220737369664 e+001$ \\
\hline 3 & $-1.574554690174881 e+003$ & $-2.512930758906020 \mathrm{e}+001$ \\
\hline 2 & $-2.972034520574520 e+004$ & $-2.973928502237764 e+004$ \\
\hline 1 & $4.364987515471394 e+0.04$ & $4.943425510576053 e+002$ \\
\hline 0 & $8.379592391659319 e+005$ & $8.379394293561344 e+005$ \\
\hline
\end{tabular}

Table 1: Interpolating function coefficients.

domain data taken together is inconsistent with the $a$ priori information, but where each class of data is by itself compatible with it.

The main result of the paper shows that the problems of establishing consistency of the data and of obtaining a nominal model and bounds on the identification error can be recast as a LMI feasibility problem that can be efficiently solved.

Additionally, we have shown that in this context the set of models consistent with both the a priori and $a$ posteriori information can be parametrized as a LFT of the experimental data, thus justifying the combination of the proposed algorithm with standard robust control synthesis techniques.

Finally, as we indicated in section III, there are still degrees of freedom available in the problem. This raises the interesting possibility of using these degrees of freedom to optimize an additional performance criteria, for instance minimizing the order of the nominal model.

Acknowledgements- The work of the first and last authors was supported by UBACyT projects IN018 and IN049, University of Buenos Aires. Mario Sznaier was supported in part by NSF under grant ECS-9211169.

\section{References}

[1] J. Ball, I. Gohberg and L. Rodman, Interpolation of Rational Matrix Functions, Operator Theory: Advances and Applications, Vol. 45, Birkhäuser, 1990. [2] S. Boyd, L. El Ghaoui, E. Feron and V. Balakrishnan, Linear Matrix Inequalities in Systems and Control Theory. SIAM Studies in Applied Mathematics, Vol 15, SIAM, Philadelphia, 1994.

[3] J. Chen, C. Nett and M. Fan, Worst-Case System Identification in $\mathcal{H}_{\infty}$ : Validation of Apriori Information, Essentially Optimal Algorithms, and Error Bounds. Proceedings American Control Conference, Chicago, IL, 1992.

[4] J. Chen, J. Farrell, C. Nett and K. Zhou, $\mathcal{H}_{\infty}$ Identification of Multivariable systems by tangential interpolation methods. Proceedings of the Conference on Decision \& Control, 1994.

[5] J. Chen and C. Nett, The Carathéodory-Fejér problem and the $\mathcal{H}_{\infty} / \ell_{1}$ identification: A time domain approach. IEEE Transactions Automatic Control, Vol. 40, No. 4, 1995.

[6] G. Gu and P. Khargonekar, A class of Algorithms for Identification in $\mathcal{H}_{\infty}$. Automatica, Vol. 28, No. 2, 1992.

[7] J. Helmicki, C. Jacobson and C. Nett, Control Oriented System Identification: A worst case/deterministic approach in $\mathcal{H}_{\infty}$. IEEE Transactions Automatic Control, Vol. 36, No. 10, 1991.

[8] C. Jacobson, C. Nett and J. Partington, WorstCase System Identification in $\ell_{1}$ : Optimal algorithms and Error Bounds. System \& Control Letters, No. 19, 1992.

[9] P. Mäkilä, Identification of stabilizable systems: closed-loop approximation. International Journal of Control, Vol. 54, No. 3, 1991.

[10] P. Mäkilä, Robust Identification and Galois sequences. International Journal of Control, Vol. 54, No. $5,1991$.

[11] P. Mäkilä, Worst-case input-output identification. International Journal of Control, Vol. 56, No. 3, 1992.

[12] M. Milanese, Worst-case $\ell_{1}$ identification. Bounding Approaches to System Identification, Milanese, Norton, Piet-Lahanier \& Walter, Editors. Plenum Press, 1994.

[13] Y. Nesterov and A. Nemirovski, Interior Point Polynomial Methods in Convex Programming: Theory and Applications. SIAM Studies in Applied Mathematics, Vol 13, SIAM, Philadelphia, 1994.

[14] J. Partington, Robust Identification in $\mathcal{H}_{\infty}$. Journal of Mathematical Analysis and Applications, Vol. 166, pp. 428-441, 1992.

[15] H. Rotstein, A Nevanlinna-Pick approach to time domain Constrained $\mathcal{H}_{\infty}$ control. To appear at the SIAM Journal of Control and Optimization.

[16] R. Sánchez Peña and C. Galarza, Practical Issues in Robust Identification. IEEE Transactions in Control Systems Technology, Vol. 2, No. 1, 1994.

[17] R. Smith, and J. Doyle, Model Validation: A Connection Between Robust Control and Identification. IEEE Transactions on Automatic Control, Vol. 37, No.7, 1992.

[18] T. Zhou and H. Kimura, Time Domain Identification for Robust Control. System \& Control Letters, Vol. 20, pp. 167-178, 1993.

[19] T. Zhou and H. Kimura, Structure of Model Uncertainty for a Weakly Corrupted Plant. IEEE Transactions on Automatic Control, Vol. 40, No. 4, 1995. 